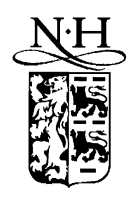

\title{
Magnetic force microscopy study of a defect-induced orthogonal magnetic structure on a cobalt film
}

\author{
Heh-Nan Lin ${ }^{\mathrm{a}, *}$, Ching-Ray Chang ${ }^{\mathrm{b}}$, Yuh-Hwang Chiou ${ }^{\mathrm{c}}$ \\ ${ }^{a}$ Precision Instrument Development Center, National Science Council, 20 R\&D Rd. VI, Science Based Industrial Park, \\ Hsinchu 300, Taiwan, ROC \\ ${ }^{\mathrm{b}}$ Department of Physics, National Taiwan University, Taipei 106, Taiwan, ROC \\ ${ }^{\mathrm{c}}$ Department of Electrophysics, National Chiao Tung University, Hsinchu 300, Taiwan, ROC
}

\begin{abstract}
An unusual orthogonal magnetic structure is observed on a cobalt film by magnetic force microscopy. The structure is composed of a double wall, which is caused by a surface scratch, and two perpendicular spike domains, which are caused by an internal defect. The occurrence of the structure is attributed to the generation of surface free poles due to film inhomogeneities and their redistribution to reduce the magnetostatic energy. The magnetization pattern has also been proposed to explain the observed magnetic force image. (C) 2000 Elsevier Science B.V. All rights reserved.
\end{abstract}

Keywords: Magnetic force microscopy; Double wall; Spike domain; Defects

Magnetic force microscopy (MFM) [1,2] has been used extensively in recent years for the investigation of magnetic domains on thin films [3-6] because of its high resolution, ease of use, and simultaneous measurement of topography. Potentially MFM should also be a useful tool for the observation of domain pinning on surface scratches or the detection of internal defects. However, experimental works on this subject are rarely reported. Here we use MFM to study the influence of a surface scratch and an internal defect on a Co film. We have observed an unusual orthogonal magnetic structure composed of a double wall and two spike domains due to surface inhomogeneities. The magnetization pattern based on the obtained image is also given.

The sample was a $115 \mathrm{~nm}$ Co film sputtered on a glass substrate in a base pressure around $10^{-6}$ Torr. The experiment was performed on a MultiMode Nanoscope III made by Digital Instruments. A pyramidal silicon tip coated with a $40 \mathrm{~nm} \mathrm{Co} / \mathrm{Cr}$ alloy film was used for experiment. The oscillation phase shift $\Delta \theta$ was recorded

\footnotetext{
*Corresponding author. Tel.: + 886-3-5779911; fax: 886-35773947.

E-mail address: hnlin@pidc.gov.tw (H.-N. Lin)
}

as the magnetic force image. The tip was magnetized in a direction normal to the film surface prior to measurement. Consequently, a strong contrast in the image implies high stray field in the same direction with bright (dark) contrast representing repulsive (attractive) force. The details of sample preparation and imaging process can be found elsewhere [6].

We first use atomic force microscopy (AFM) to identify an area with notable surface scratches and then obtain an MFM image on the same area. In Fig. 1, a $40 \times 40 \mu \mathrm{m}^{2}$ scan is presented. Fig. 1a shows the morphology, where a lot of scratches are observed. Fig. $1 \mathrm{~b}$ is the simultaneously acquired magnetic force image, where two parallel cross-tie walls and an unusual orthogonal magnetic structure in between are observed. The cross-tie walls have rhomb structures with alternating bright and dark contrasts inside. The detailed examination of the properties of cross-tie walls can be found elsewhere $[5,6]$.

The orthogonal structure located in the center is composed of a double wall and two spike domains perpendicular to the double wall. Although the contrasts are not obvious on some locations, the double wall has dark and bright contrast from left to right along the two single walls, which have a separation of around $700 \mathrm{~nm}$. By a careful inspection of Fig. 1a, we find a shallow but 

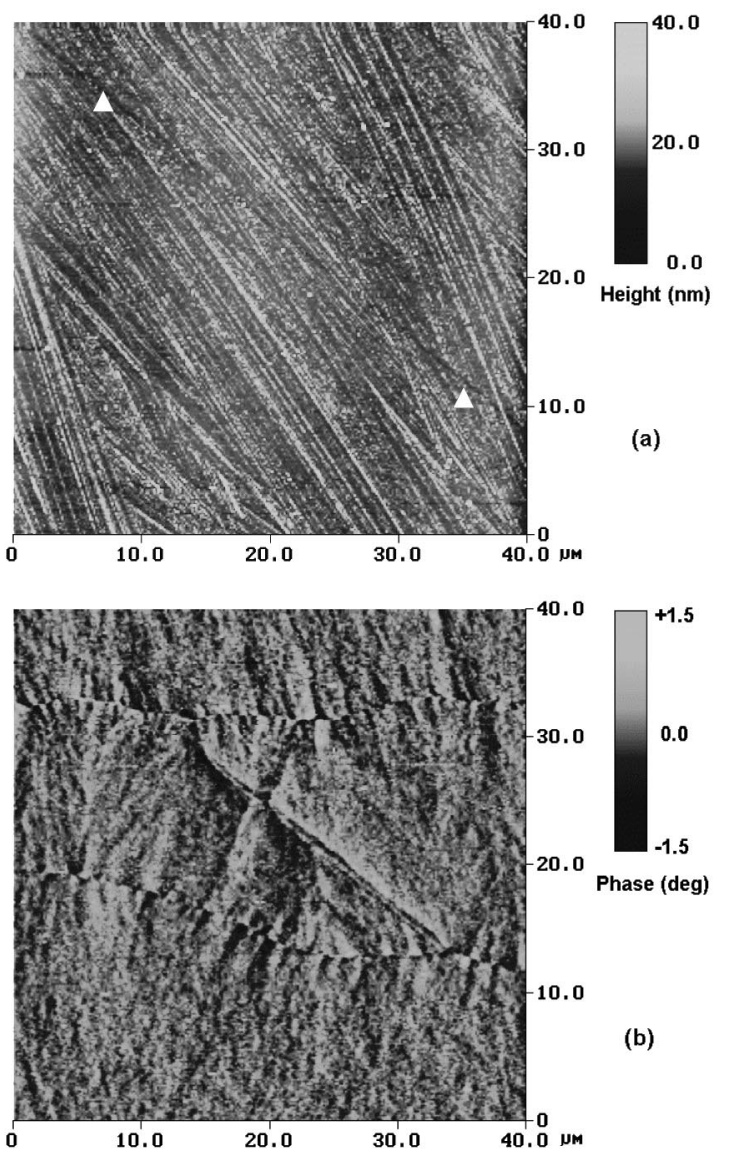

Fig. 1. (a) Topographic image of the $115 \mathrm{~nm}$ Co film, and (b) magnetic force image on the same area. The scan size is $40 \times 40 \mu \mathrm{m}^{2}$. The triangles in (a) mark the scratch.

recognizable scratch (marked by triangles) extending across the two parallel cross-tie walls at exactly the same location of the double wall. The magnetization pattern along the scratch is depicted in Fig. 2. Since a scratch is usually accompanied with bumps on the edge, the magnetic poles resulting from the termination of the in-plane magnetization will have different polarities and densities on each side of the scratch across the bumps (as in the inset of Fig. 2). The magnetic flux emerging from the positive poles will end at the negative poles and thus will produce a double wall as seen in Fig. $1 b$.

The two spike domains at first sight resemble a single rhomb in the cross-tie wall if the size is not taken into account. Indeed, a large rhomb was observed in our previous study [6]. Nevertheless, the two domains separated by the double wall have the same shape and contrast except they are shifted by a distance of around $1.4 \mu \mathrm{m}$. This is distinctively different from the antisymmetric contrast associated with the rhombs occurring in a cross-tie wall. Actually, spike domains were first theoretically dis-

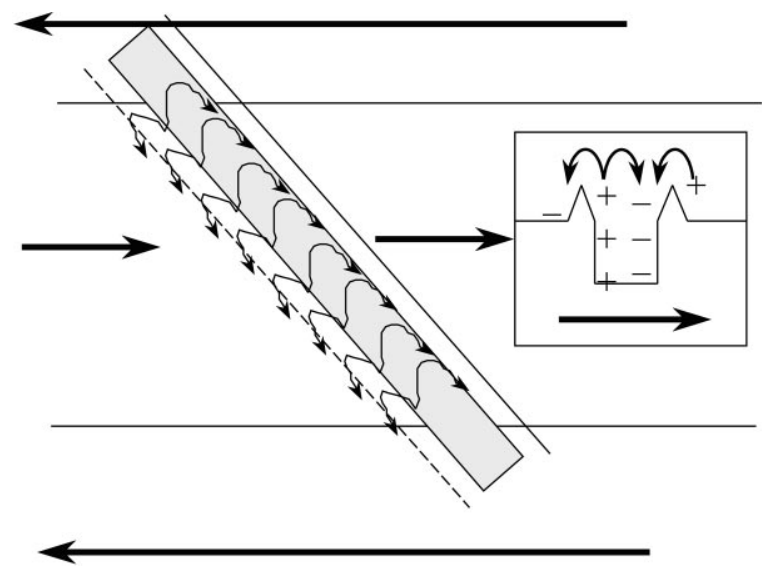

Fig. 2. The schematic diagram of magnetic flux and free poles around a single surface scratch. The inset is the cross-section of the scratch and the distribution of the poles. Curved arrows represent the magnetic flux and the straight arrows are magnetization.

cussed by Néel in 1944 and experimentally observed later [7]. Their appearance is attributed to surface defects (or inclusions), which can be simply considered as nonmagnetic regions. Because of the distinctive spike-shape, we believe an internal defect exists near the center although there is no trace of defects in Fig. 1a.

When a defect is present in a domain, magnetic free poles generated at edges of the defects will usually redistribute to a larger area to reduce the magnetostatic energy (Fig. 3). The spike domains, which interrupt the double wall and possess dark-bright triangular shapes on each side of the double wall, are the result of such redistribution of free poles. It should be noted that the observed spike domains in Fig. 1b have a complicated magnetic flux distribution as shown in Fig. 3b. The pole distribution diagrams in Fig. 3 have been drawn to demonstrate the effect of the defect. If we apply Gauss' theorem to one edge of the defect (or inclusion), the total amount of free poles is given by the product of the normal component of the magnetization and the crosssection area of the defect and scratch. Therefore, the total amount of magnetic poles is exactly the same in Fig. 3a and $3 \mathrm{~b}$ as in Gauss' theorem. An important result of Fig. $3 b$ is that the magnetic free poles have opposite polarities at the edges of each spike. Such a feature cannot be distinguished in a Bitter pattern since it only exhibits field intensity at the edges [7], but has also been clearly resolved by Kerr and Lorentz microscopies [8,9].

The above description may raise the question, why the specific scratch only induces the observed double wall but not others. This can be explained by the existence of the defect near the scratch since the amount of magnetic free poles is then substantially increased and a strong contrast thus appears in the image. Also, it may be 

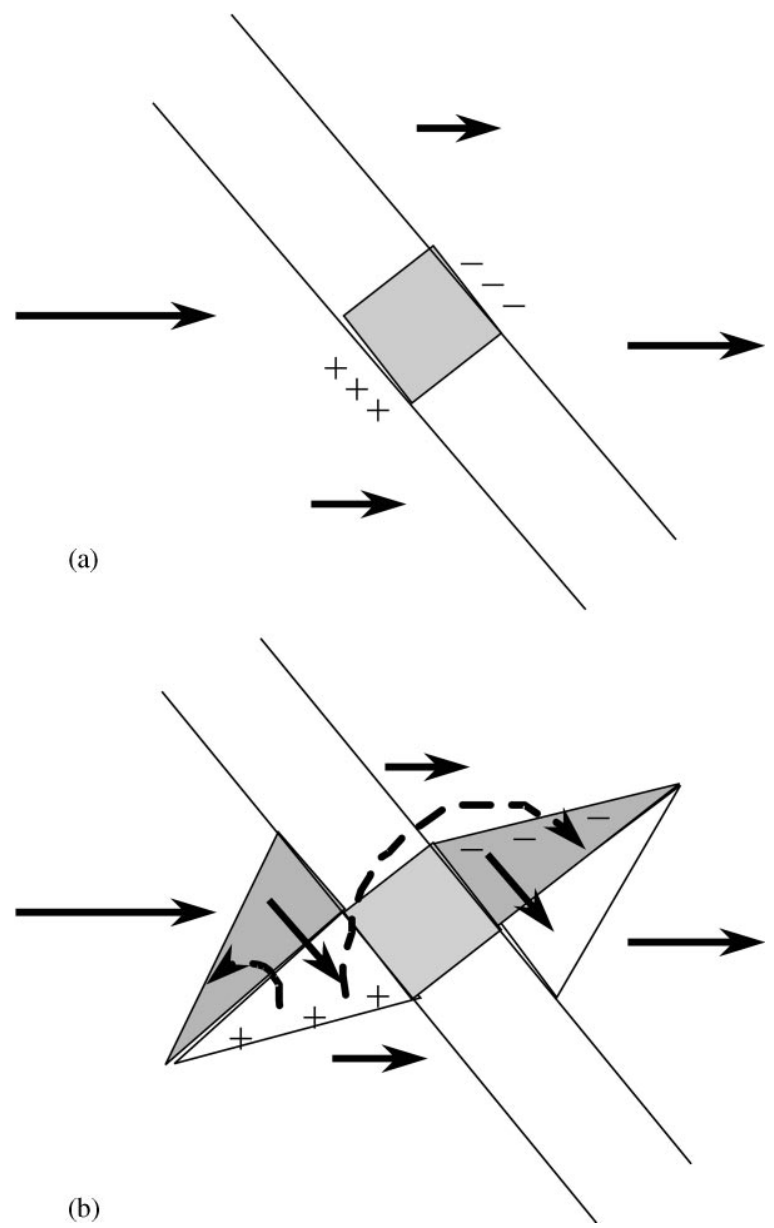

Fig. 3. The schematic diagrams of magnetic flux and free poles around a defect (a) without domains and (b) with spike domains. Curved arrows represent the magnetic flux and the straight arrows are the magnetization. argued that the whole orthogonal structure is caused by the defect only and the scratch has no effect. Previous experimental results, however, showed only two spike extensions around a defect without any double walls [7-9]. Therefore, the present interpretation should be plausible.

In conclusion, we have observed an unusual orthogonal magnetic structure between two cross-tie walls on a Co film. We interpret the resulting structure as caused by a surface scratch and an internal defect. The double wall and the spike domains are formed to reduce the magnetostatic energy. Even though the surface scratch is only vaguely observed in the AFM picture, the MFM image clearly designates the position of the surface scratch and the internal defect. The present study suggests a possible nondestructive method of locating surface scratch or internal defect in a ferromagnetic film.

\section{References}

[1] Y. Martin, H.K. Wickramasinghe, Appl. Phys. Lett. 50 (1987) 1455.

[2] K. Babcock, M. Dugas, S. Manalis, V. Elings, Mater. Res. Soc. Symp. Proc. 355 (1995) 311.

[3] R.B. Proksch, S. Foss, E.D. Dahlberg, IEEE Trans. Magn. 30 (1994) 4467.

[4] M. Hehn, S. Padovani, K. Ounadjela, J.P. Bucher, Phys. Rev. B 54 (1996) 3428.

[5] M. Löhndorf, A. Wadas, H.A.M. van den Berg, R. Wiesendanger, Appl. Phys. Lett. 68 (1996) 3635.

[6] H.-N. Lin, Y.H. Chiou, B.-M. Chen, H.-P.D. Shieh, C.-R. Chang, J. Appl. Phys. 83 (1998) 4997.

[7] B.D. Cullity, Introduction to Magnetic Materials, AddisonWesley, Reading, MA, 1972, p. 317.

[8] A. Hubert, R. Schäfer, Magnetic Domains: The Analysis of Magnetic Microstructures, Springer, New York, 1998, p. 483.

[9] A.F. Smith, J. Phys. D 3 (1970) 1044. 